\title{
Genome-wide metabolic re-annotation of Ashbya gossypii: new insights into its metabolism through a comparative analysis with Saccharomyces cerevisiae and Kluyveromyces lactis
}

\author{
Daniel Gomes, Tatiana Q Aguiar, Oscar Dias, Eugénio C Ferreira, Lucília Domingues and Isabel Rocha*
}

\begin{abstract}
Background: Ashbya gossypii is an industrially relevant microorganism traditionally used for riboflavin production. Despite the high gene homology and gene order conservation comparatively with Saccharomyces cerevisiae, it presents a lower level of genomic complexity. Its type of growth, placing it among filamentous fungi, questions how close it really is from the budding yeast, namely in terms of metabolism, therefore raising the need for an extensive and thorough study of its entire metabolism. This work reports the first manual enzymatic genome-wide re-annotation of A. gossypii as well as the first annotation of membrane transport proteins.

Results: After applying a developed enzymatic re-annotation pipeline, 847 genes were assigned with metabolic functions. Comparatively to KEGG's annotation, these data corrected the function for $14 \%$ of the common genes and increased the information for 52 genes, either completing existing partial EC numbers or adding new ones. Furthermore, 22 unreported enzymatic functions were found, corresponding to a significant increase in the knowledge of the metabolism of this organism. The information retrieved from the metabolic re-annotation and transport annotation was used for a comprehensive analysis of A. gossypii's metabolism in comparison to the one of S. cerevisiae (post-WGD - whole genome duplication) and Kluyveromyces lactis (pre-WGD), suggesting some relevant differences in several parts of their metabolism, with the majority being found for the metabolism of purines, pyrimidines, nitrogen and lipids. A considerable number of enzymes were found exclusively in A. gossypii comparatively with K. lactis (90) and S. cerevisiae (13). In a similar way, 176 and 123 enzymatic functions were absent on A. gossypii comparatively to K. lactis and S. cerevisiae, respectively, confirming some of the well-known phenotypes of this organism.
\end{abstract}

Conclusions: This high quality metabolic re-annotation, together with the first membrane transporters annotation and the metabolic comparative analysis, represents a new important tool for the study and better understanding of A. gossypii's metabolism.

Keywords: Genome re-annotation, Ashbya gossypii, Metabolic functions, Yeast metabolism

\section{Background}

Ashbya gossypii (syn. Eremothecium gossypii) is a filamentous fungus that has long been known mainly as a riboflavin overproducer. Its relatively simple life cycle in the laboratory, together with the astonishing similarity of its genome with the genome of the yeast Saccharomyces

\footnotetext{
*Correspondence: irocha@deb.uminho.pt

CEB - Centre of Biological Engineering, Universidade do Minho, Campus de Gualtar, 4710-057 Braga, Portugal
}

(c) 2014 Gomes et al.; licensee BioMed Central Ltd. This is an Open Access article distributed under the terms of the Creative Commons Attribution License (http://creativecommons.org/licenses/by/4.0), which permits unrestricted use, distribution, and reproduction in any medium, provided the original work is properly credited. The Creative Commons Public Domain Dedication waiver (http://creativecommons.org/publicdomain/zero/1.0/) applies to the data made available in this article, unless otherwise stated.

cerevisiae, have made this fungus an attractive biological model for fungal developmental studies (reviewed in Wendland and Walther [1], Schmitz and Philippsen [2]). Based on the gene order, 91\% of the 4776 annotated $A$. gossypii's genes are syntenic and only $4 \%$ non-syntenic to S. cerevisiae genes $[2,3]$. The remaining $5 \%$ have no homologues in S. cerevisiae [3].

The potential of A. gossypii as a host for the production of heterologous proteins has been recently investigated, 
through the expression of two cellulases from Trichoderma reesei, endoglucanase I and cellobiohydrolase I, and an Aspergillus niger $\beta$-galactosidase [4,5]. A. gossypii possesses the capacity to secrete heterologous enzymes to the extracellular medium and to recognize signal peptides from other organisms as secretion signals $[4,5]$, which is a desired property for cost-efficient downstream processing of low- and medium-value enzymes. Also, it is able to perform post-translation protein modifications, such as $N$-glycosylation, and other modifications required for biological activity and protein stability [4-6]. Compared to the closely related yeast $S$. cerevisiae, one of the fungal hosts most commonly used for the production of heterologous proteins $[7,8]$, A. gossypii seems to have the tendency to hyperglycosylate less extensively (especially its $N$-glycans), which is beneficial for the production of proteins whose properties may be adversely affected by extensive glycosylation $[4,6]$.

These features, combined with the availability of several tools for $A$. gossypii's easy genetic manipulation, have raised attention to this fungus as a potential cell factory organism, which could be tailor-made to produce other metabolites and/or proteins. One possible strategy that may assist with this task is the application of Systems Biology tools. Genome-Scale Metabolic Models (GSMMs) are among the most employed tools within this field. Based on the genome of an organism and on relevant physiological data, the full set of metabolic functions is assembled in a metabolic network that can be converted to the correspondent mathematical model. Central to these models is the stoichiometric matrix which covers all the metabolites and reactions present in the metabolism of the organism. Optimization tools can then be applied to these models for different purposes, such as simulate cell growth on different media, the impact of genetic engineering strategies in the phenotype or to analyze the robustness of the network [9].

The initial element in the re-construction of a genomescale metabolic model is to collect data from the genome annotation [10], from which the enzymatic functions putatively coded within the entire genome can be collected. Such information can be consulted in non organism-specific databases, such as NCBI [11], or organism-specific databases, such as the Saccharomyces Genome Database (SGD; [12]) for $S$. cerevisiae or the Ashbya Genome Database (AGD; [13]) for A. gossypii.

The first genome annotation of $A$. gossypii was published in 2004 following its sequencing process [3]. This information corresponded to an association of A. gossypii genes to possible homologues, based on sequence similarity data. Subsequently, AGD came as a comprehensive online database presenting different information for $A$. gossypii genes based on sequence similarity and synteny degree with closely-related organisms [13]. However, despite presenting Gene Ontology (GO) data for most of $A$. gossypii genes, the information existent on AGD fails to provide a clear enzymatic functional annotation for these genes, which is usually translated in the form of EC numbers. Additionally, the genomic information for a specific microorganism is constantly evolving, as an outcome of the continuous development of bioinformatics tools, as well as the generation of new experimental data, which may allow the discovery of new proteins and genes [14] or change the information reported about them. For this reason, a process of genome re-annotation may represent a critical element to obtain reliable and updated information for a given organism. According to Ouzounis and Karp [15], an average of $7 \%$ of the genes are assigned with new functions in a re-annotation process, which represents a considerable amount of new information generated on these processes.

The functional re-annotation of an entire genome can be a very time consuming process, according to the size of the genome and the criteria applied. Possibly for this reason, only few genome-wide re-annotations were reported until now, including for Campylobacter jejuni NCTC11168 [16], Mycobacterium tuberculosis H37Rv [17], Escherichia coli [18], Bacillus subtilis [19], Arabidopsis thaliana [20] and, more recently, Kluyveromyces lactis [14]. To increase the speed of the process, some computational tools can be used to facilitate this task [21]. However, the probability of an error increases as manual curation contribution is smaller.

Here we describe a thoroughly curated genome-wide enzymatic functional re-annotation for $A$. gossypii and the first membrane transport proteins annotation for this organism. With the aim of getting further insights into A. gossypii's metabolism, a comparative analysis was conducted between the metabolic capabilities of $A$. gossypii and those of $S$. cerevisiae and $K$. lactis, as these are organisms phylogenetically close to A. gossypii [22] and for which there is updated information available in the literature or in databases ([14]; SGD).

\section{Methods}

\section{Re-annotation sources}

Source A: UniProtKB/Swiss-Prot [23,24] refers to a particular section of UniProtKB that presents manually annotated and reviewed information.

Source B: Saccharomyces Genome Database (SGD $[12,25])$ refers to information with a high degree of reliability, ensured by a team of biocurators who frequently review the information available in this platform.

Source C: Ashbya Genome Database (AGD - [13,26]) refers to a comprehensive database containing different genomic information for $A$. gossypii based on sequence similarity and synteny degree comparatively to other organisms. 
Source D: UniProtKB/TrEMBL [23,24] refers to the non-reviewed section of UniprotKB.

Source E: merlin [14,21] (available at http://www.merlin-sysbio.org/) refers to an in-house developed software tool that, among other features, provides homology analyses against a wide range of organisms using different tools.

\section{Other databases used}

Transporter Classification DataBase (TCDB - $[27,28]$ ) is a database that details a classification system for membrane transporter proteins, the Transporter Classification (TC) system.

Kyoto Encyclopedia of Genes and Genomes (KEGG $[29,30])$ is a database presenting information of the biological systems on different levels, ranging from organism related information, such as genome annotations, to enzymes specifications or metabolites information.

BRENDA [31,32] is a database that gathers a very wide range of enzymes information mostly based on experimental data.

PEDANT 3 [33,34] is a database that presents several automatic analyses for a high amount of protein sequences. These analyses are performed based in several bioinformatics tools, which allow a wide characterization of a given protein.

\section{Pipeline for the functional re-annotation of the A. gossypii's genome}

The A. gossypii's ATCC 10895 genome was retrieved from NCBI [11] in the form of FASTA files and loaded into merlin [14,21]. For each gene, this tool performed remote Basic Local Alignment (BLAST; [35]) and Hidden Markov Models (HMMs; [36]) searches against NCBI databases (all non-redundant GenBankCDS) to find possible homologues in other species. BLAST searches were conducted using the program blastp, the matrices BLOSUM62 and PAM30, and a maximum e-value of $1 \mathrm{E}-30$. Both searches were restricted to a maximum of 100 homologues. Based on the taxonomy of the organism and the number of similar hits, a confidence score (C.S.) is calculated to each possible homologue (for a detailed description see Dias et al. [21]). For this purpose, a $\alpha$ value of 0.3 was adopted on this case. If at least one possible homologue is found for a given gene (independently of the C.S.) that presents, at least, one EC number associated, that gene is considered a putative metabolic gene and the re-annotation pipeline is applied (Figure 1).

The re-annotation pipeline consists in a systematic manual process to find and collect information from different sources, enabling the assignment of one or multiple enzymatic functions to a given putative metabolic gene. Depending on the used source, the function(s) assigned to each gene had different levels of reliability. The most reliable information was retrieved from UniProtKB/SwissProt, as this database contains manually curated and analyzed data. The total number of entries obtained through this source was, however, considerably small comparatively with other sources and insufficient to cover the entire genome of $A$. gossypii. To surpass this limitation, the current pipeline defines the utilization of five different information sources (Figure 1A). The utilization of these sources was defined by a decision-making structure that aimed at promoting, for every re-annotated gene, the utilization of the source with the highest reliability. To enable the collection of the most complete information possible, different databases were consulted when trying to collect a full EC number for the metabolic genes (Figure 1B).

A detailed description of the re-annotation pipeline is provided on Additional file 1.

\section{Annotation of membrane transporter proteins}

In addition to the enzymatic functional re-annotation, membrane transporters were also sought in the A. gossypii's genome. For that purpose, a recently in-house developed tool was employed, which allows the identification and characterization of the membrane transporter systems coded within a specific genome [37]. This tool enables to define in detail the specific transport reaction(s) that may be associated to a given gene, including the transported metabolites, reversibility, co-factor/energy usage, location on the cell, etc.. In this work, the tool was only employed to perform the initial phase of the transporter systems annotation, which consists on the assignment of a TC (Transporter Classification) number (or more than one) to putative membrane transporter-coding genes.

In an initial step, transporter candidate genes (TCGs) were identified applying a TMHMM (TransMembrane prediction using Hidden Markov Models) search over the entire genome [38], which allows predicting transmembrane helices within the several proteins coded by the genome. The presence of one putative transmembrane helice classifies the gene as a TCG, making it eligible for the second step of this process. Here, SW (Smith-Waterman) local alignments [39] are performed over the entire set of TCGs against the TCDB [27]. These alignments allow the identification of genomecoded proteins with a strong sequence similarity to TCDB entries, considering a specific similarity threshold. The standard similarity threshold is set on $10 \%$ of identity, but it can be reduced through the application of a heuristic method that considers the number of transmembrane helices initially identified. Based on the sequence similarities found for each gene, one (or multiple) TC number could be assigned to that gene. A detailed description of this tool is provided by Dias [37]. 


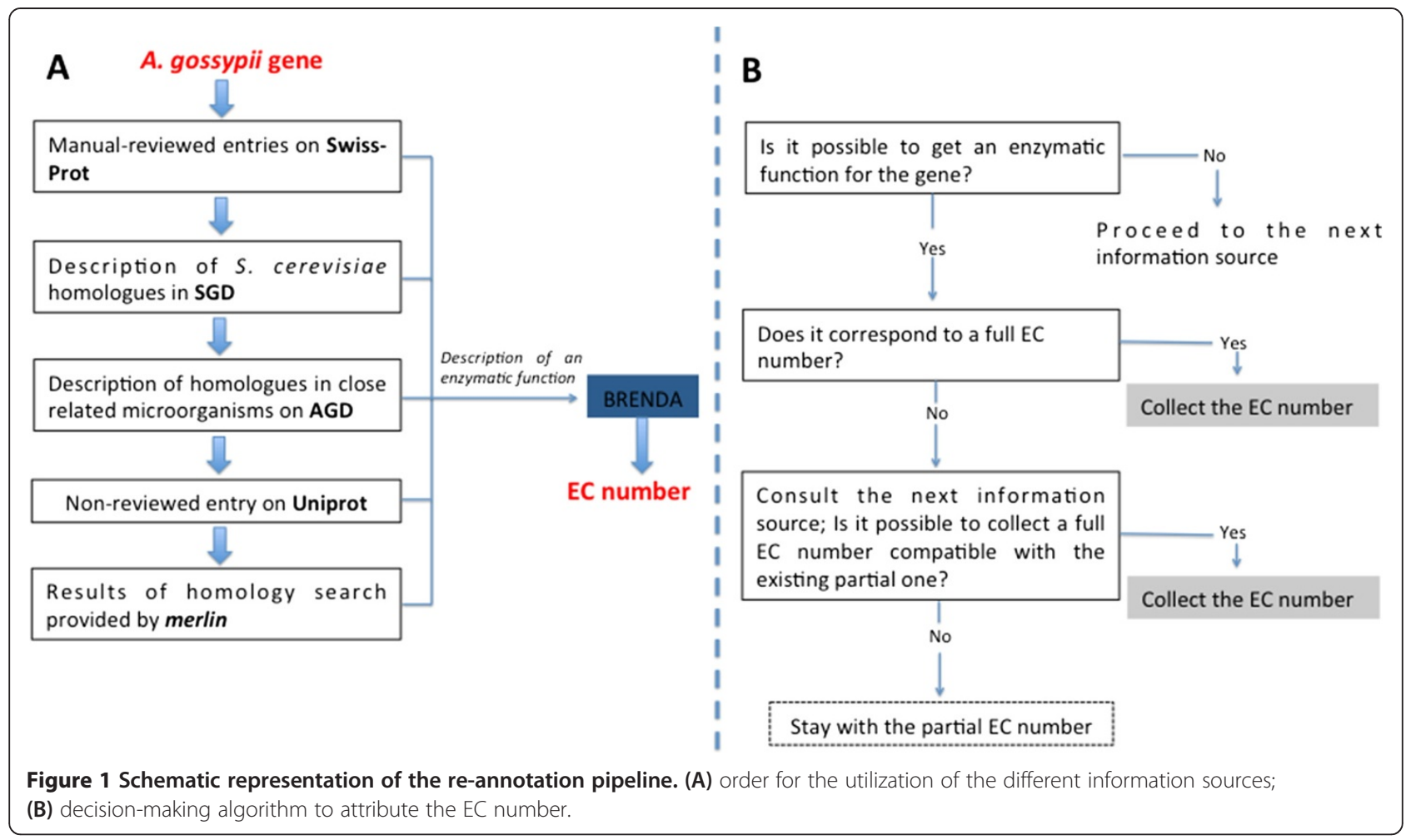

\section{Classification of a gene or an enzyme into a pathway}

To classify a gene regarding its metabolic role in the cell, the KEGG's internal classification system for pathways was used, taking either the classification for the gene or, if not available, the classification for the EC numbers associated to that gene. For enzyme classification, a similar strategy was adopted, consulting firstly the pathways classification on the KEGG's page of the enzyme and, if not available, the classification provided by KEGG for associated genes.

An additional classification system was still used for the enzymes and genes with no pathway(s) assigned from KEGG, based on the FunCat nomenclature [40]. For that, the biological functions assigned to the genes that were associated to a given enzyme were retrieved from the PEDANT 3 database [33].

\section{Results and discussion}

In this work, two main annotation processes were performed: a whole-genome functional metabolic reannotation and the first annotation of membrane transport proteins for the fungus A. gossypii.

After applying the functional re-annotation pipeline described in the Methods section, a total of 847 genes were annotated with an EC number. Among these, 777 genes were associated with only one EC number (monofunctional genes) and 70 genes were annotated with more than one: 52 genes with 2 ECs, 11 genes with 3, 4 genes with 4, 2 genes with 5 , and 1 gene with 7 . Regarding the multifunctional genes, 22 presented EC numbers from different enzymatic families. One of these cases was the gene AGOS_AFR703W, which by homology with HIS4 from S. cerevisiae was annotated as one oxidoreductase (1.1.1.23) and two hydrolases $(3.5 .4 .19,3.6 .1 .31)$, all involved in histidine biosynthesis. Another class of genes was observed that may help to understand some of the events associated to the separation of S. cerevisiae and A. gossypii. According to Brachat et al. [41], one of the consequences of those events was the distribution of multiple functions over different genes that were before in a multifunctional gene, which constitutes the sub-functionalization model of evolutionary divergence of duplicated genes. One of these cases is possibly AGOS_AFR234W presenting two homologues in S. cerevisiae with distinct functions. According to SGD, YGL224C (SDT1) codes for a pyrimidine nucleotidase while YER037W (PHM8) codes for a lysophosphatidic acid phosphatase.

It is worth noting that full EC numbers were given to $92.0 \%$ of the annotated genes, which constitutes an advantage in the context of a metabolic model reconstruction process. Partial EC numbers cannot be directly used on this process as they represent non-specific information, impairing the elaboration of a clear biochemical reactions set.

The distribution of the annotated genes by enzymatic family, depicted in Table 1, was considerably heterogeneous. Transferases was clearly the enzymatic family with the highest representation among the annotated genes, 
Table 1 Distribution of re-annotated genes over the different enzymatic families

\begin{tabular}{lcc}
\hline Enzymatic family & $\begin{array}{c}\text { Number of genes } \\
\text { annotated }\end{array}$ & $\begin{array}{c}\text { \% Annotated } \\
\text { genes }\end{array}$ \\
\hline Oxidoreductases (1.-) & 195 & 23.0 \\
Transferases (2.-) & 295 & 34.8 \\
Hydrolases (3.-) & 221 & 26.1 \\
Lyases (4.-) & 65 & 7.7 \\
Isomerases (5.-) & 26 & 3.1 \\
Ligases (6.-) & 72 & 8.5 \\
\hline
\end{tabular}

with $34.8 \%$ of the genes. That was followed by Hydrolases, Oxidoreductases, Ligases, Lyases and Isomerases.

Regarding the annotation of membrane transport proteins, the first reported to date for A. gossypii, a total of 265 genes were annotated with one or multiple TC number(s), belonging to one of the seven families of membrane transport proteins described on TCDB. The distribution of genes over the different transporter families (Table 2) was compared to the one reported by Dias et al. [14] for K. lactis and Resende et al. [42] for H. pylori, which were obtained using the same tool [37]. From this comparison it was possible to observe that for these three different microorganisms, the transporter class 1.- 2.- and 3.- presented the highest number of associated genes, suggesting an important role of these type of transporters on the cell.

\section{Characterization of the re-annotation sources}

The enzymatic functional re-annotation performed in this work was based on different sources consulted on a top-to-bottom way in terms of reliability. Some types of information, like those originated from Class A (see Re-annotation sources in the section Methods), have a high degree of confidence as they were obtained from manually reviewed and curated sources. Other classes, however, provided information not manually curated and, therefore, less reliable. By evaluating the type of data employed on the re-annotation process, the reliability of the re-annotation results could be assessed.
As can be observed in Figure 2, the main source used in the re-annotation process was the SGD, contributing for $75 \%$ of the re-annotated genes. Although this source does not provide a direct functional annotation, as Swiss-Prot does, but instead an annotation by homology, it still presents a high level of reliability, since the information retrieved from SGD is manually curated and analyzed. With a much lower, but still significant number of genes, Swiss-Prot contributed for the re-annotation of $19 \%$ of the re-annotated genes. The contribution of these two highly-reliable sources for the re-annotation of nearly $94 \%$ of the genes supports a consistent re-annotation process.

\section{Other available annotations for A. gossypii}

The first complete annotation of the A. gossypii's genome was publicly released in 2004 [3]. At the time, 4718 genes were annotated as protein-coding genes, but since then new protein-coding loci have been identified and the initial annotation improved. In 2007, Gattiker et al. [13] released the AGD 3.0, which included updated DNA annotation and microarray RNA expression data for A. gossypii. Subsequently, revised annotations of the reported ORFs in A. gossypii have been regularly updated in the NCBI GenBank database, with the latest version dating from August of 2012.

The current work provides the first manually curated whole-genome annotation of $A$. gossypii's metabolic functions. This corresponds to the first set of curated data that directly attributes an EC number to each putative metabolic gene on $A$. gossypii. Different databases, such as BRENDA or Uniprot, provide some degree of annotation; however, they are limited to a small number of genes, insufficient for wider approaches, such as for a genomescale metabolic model reconstruction. On the other hand, KEGG presents a very complete annotation and can, thus, serve for comparison purposes with our re-annotation. KEGG is a complex database covering multiple levels of the biological systems (genes, pathways and compounds). In what concerns gene annotation, a wide range of organisms are covered, from entire to partial genomes. For the majority of the cases, this information is, however,

Table 2 Distribution of annotated genes over the different membrane transport proteins classes

\begin{tabular}{|c|c|c|c|c|}
\hline Transporter protein class & Number of annotated genes & $\%$ Annotated genes & $\begin{array}{c}\% \text { Annotated } \\
\text { genes - K. lactis }\end{array}$ & $\begin{array}{c}\% \text { Annotated } \\
\text { genes }-H . \text { pylori }\end{array}$ \\
\hline 1. Channels/pores & 35 & 13.2 & 9.6 & 13.7 \\
\hline 2. Electrochemical potential-driven transporters & 141 & 53.2 & 65.4 & 35.8 \\
\hline 3. Primary active transporters & 80 & 30.2 & 15.3 & 43.5 \\
\hline 4. Group translocators & 0 & - & 0.3 & 0.7 \\
\hline 5. Transmembrane electron carriers & 3 & 1.1 & - & - \\
\hline 8. Accessory factors involved in transport & 0 & - & 1.3 & - \\
\hline 9. Incompletely characterized transport systems & 21 & 7.9 & 8.0 & 6.1 \\
\hline
\end{tabular}




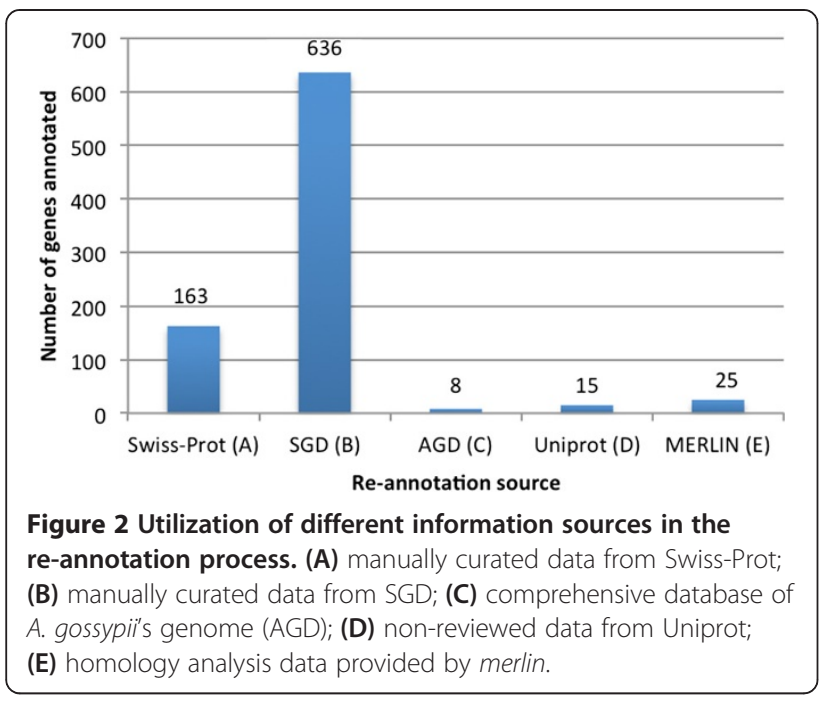

generated automatically from GenBank data with computational tools [30]. KEGG provides EC number information for $1111 A$. gossypii's genes, 264 more genes than our annotation (847). A total of 668 genes are present in both annotations, and 443 and 179 genes were exclusively annotated by KEGG and the current re-annotation, respectively. Among the set of 668 common genes, 95 presented annotations that did not match. These 95 genes were analyzed and classified into four different classes (I, II, III and IV) according to the reason why the two annotations were different.

\section{Mismatches in gene annotation}

Class I, which includes 35 genes, refers to the cases where the current re-annotation allowed to complete or increase the information relatively to a partial EC number annotated by KEGG. For example, the KEGG's annotation for AGOS_ADR132W is the partial EC number 1.-...-, while our annotation for this gene is 1.16.3.1. This increase in the EC number information level may correspond to an important contribute on a posterior metabolic model reconstruction process.

Class II, with 17 genes, corresponds to the cases for which the current annotation added at least one new EC number comparatively with the KEGG's annotation. This increase in the information provided by our annotation includes not only enzymatic functions that were already present in the KEGG annotation but allocated to another gene, but also new EC numbers that were not associated in KEGG to any A. gossypii gene. As it can be seen in Table 3, among the 25 additional EC numbers associated to genes of class II, 22 were not present in the overall KEGG annotation for A. gossypii, thus representing additional enzymatic functions.

With 25 cases, class III presents those genes for which the two annotations reported different EC numbers, belonging either to the same enzymatic family or to distinct enzymatic families. One example is AGOS_ACL044W, annotated in KEGG with the EC number 1.2.1.3 and in the current annotation with 1.2.1.5. Despite different, these EC numbers are both associated to oxiredox reactions. A different example is AGOS_ADR323C, annotated in KEGG as an Isomerase (5.4.2.1), but as a Hydrolase (3.1.3.37) in the present annotation.

Finally, with 18 genes, class IV includes the cases that were re-annotated in this work with levels of information lower than those available in KEGG. All of these genes were manually revised one additional time and no evidence was found that supported the extra information on KEGG.

\section{Genes exclusively annotated by KEGG}

The 443 genes that were exclusively annotated by KEGG were analyzed to understand their exclusion from the current annotation. KEGG provides an internal classification into pathways for some of its genes/enzymes. Based on this information (see a detailed description on the section Methods) we were able to classify 323 of the 443 genes. The remaining genes were analyzed and classified using the FunCat system (c.f. the section Methods for a detailed description and the Additional file 2), but are not discussed in this work, as they refer to what is considered here as non-metabolically relevant functions. Most of the genes with an available classification from KEGG were also not associated to metabolically relevant functions, but instead to pathways associated with cell maintenance and signaling, cell cycle (e.g. mitosis and meiosis), maintenance and modification of nucleic acids or modification of proteins (ubiquitin-related or proteases) (c.f. Additional file 2). Although these are all important processes for the cell, they are not directly involved in the main metabolic processes leading to the production of relevant compounds or biomass. At most, some of these functions (like those of protein kinases) may indirectly affect the metabolism of the cell, but their influence cannot be clearly stated in the re-construction of a stoichiometric metabolic model [43].

The remaining genes, included by KEGG in putative metabolically relevant pathways (Figure 3) were distributed over a wide number of pathways. However, with the exception of only two genes (AGOS_AFR115W; AGOS_AFL046W), all of the genes were once again associated to non-metabolically relevant functions, and thus cannot be introduced in the reconstruction of a metabolic model. One of these cases corresponds to DNA-directed RNA polymerase (2.7.7.6) and DNA-directed DNA polymerase (2.7.7.7) (Purine and Pyrimidine metabolism).

\section{Genes exclusively annotated by the current re-annotation}

A total of 179 genes were exclusively annotated by the current re-annotation. The complete list of pathways 
Table 3 Genes with additional enzymatic functions on the current annotation when compared with KEGG's annotation (Class II)

\begin{tabular}{|c|c|c|c|}
\hline Gene & EC number & Product & KEGG $^{1}$ \\
\hline AGOS_ABR229C & 1.1.1.9 & D-xylulose reductase & No \\
\hline AGOS_ACR211W & 4.1.1.43 & Phenylpyruvate decarboxylase & No \\
\hline AGOS_ACR211W & 4.1.1.74 & Indolepyruvate decarboxylase & No \\
\hline AGOS_ADL071C & 3.1.3.68 & 2-deoxyglucose-6-phosphatase & Yes \\
\hline AGOS_ADL287C & 1.5.1.30 & Flavin reductase (NADPH) & No \\
\hline AGOS_ADL287C & 1.5.1.39 & FMN reductase $(\mathrm{NAD}(\mathrm{P}) \mathrm{H})$ & No \\
\hline AGOS_ADR115W & 2.1.1.64 & 3-demethylubiquinol 3-O-methyltransferase & No \\
\hline AGOS_ADR262C & 1.6.5.3 & $\mathrm{NADH}$ :ubiquinone reductase $(\mathrm{H}(+)$ translocating) & No \\
\hline AGOS_AEL091C & 5.4.99.28 & tRNA pseudouridine32 synthase & No \\
\hline AGOS_AEL091C & 3.5.4.26 & Diaminohydroxyphosphoribosylaminopyrimidine deaminase & No \\
\hline AGOS_AEL161W & 4.2.1.22 & Cystathionine beta-synthase & Yes \\
\hline AGOS_AEL301W & 3.6.3.35 & Manganese-transporting ATPase & No \\
\hline AGOS_AER085C & 1.3.1.10 & enoyl acyl-carrier-protein reductase & No \\
\hline AGOS_AER085C & 2.3.1.38 & [Acyl-carrier-protein] S-acetyltransferase & No \\
\hline AGOS_AER085C & 2.3.1.39 & [Acyl-carrier-protein] S-malonyltransferase & Yes \\
\hline AGOS_AER085C & 4.2.1.59 & 3-hydroxyacyl-[acyl-carrier-protein] dehydratase & No \\
\hline AGOS_AER085C & 1.3.1.9 & enoyl-[acyl-carrier-protein] reductase (NADH) & No \\
\hline AGOS_AER085C & 3.1.2.14 & oleoyl-[acyl-carrier-protein] hydrolase & No \\
\hline AGOS_AER401W & 1.1.1.6 & Glycerol dehydrogenase & No \\
\hline AGOS_AER401W & 1.1.1.72 & Glycerol dehydrogenase (NADP(+)) & No \\
\hline AGOS_AFR369W & 2.7.4.22 & UMP kinase & No \\
\hline AGOS_AFR548C & 2.6.1.39 & 2-aminoadipate transaminase & No \\
\hline AGOS_AGL199C & 2.7.1.82 & Ethanolamine kinase & No \\
\hline AGOS_AGR002W & 2.3.3.5 & 2-methylcitrate synthase & No \\
\hline AGOS_AGR305W & 3.5.4.26 & Diaminohydroxyphosphoribosylaminopyrimidine deaminase & No \\
\hline AGOS_AGR344W & 2.7.8.1 & Ethanolaminephosphotransferase & No \\
\hline
\end{tabular}

${ }^{1}$ refers to the presence of the specific EC number on KEGG's annotation.

associated to this set of genes is considerably extensive (c.f. Additional file 2), covering a wide portion of the $A$. gossypii metabolism. Figure 4 shows the metabolic pathways with the highest number of associations among the analyzed set of genes, where oxidative phosphorylation clearly shows up as the pathway with the highest number. After inspecting some of these genes, it was found that in many cases a descriptive annotation was available in KEGG, even though no EC number was provided. Thus, some of the genes presented on Figure 4 are not completely absent from the KEGG annotation.

Metabolic capabilities of $A$. gossypii and comparison with S. cerevisiae and $K$. lactis

Two widely studied features of the fungus $A$. gossypii are its polarized filamentous growth and its natural riboflavin over-producing capacity. While the first has been mostly associated to regulatory mechanisms [44], the second can also be associated to the metabolic particularities of this organism. The high level of gene homology and gene order conservation between $A$. gossypii and the yeast $S$. cerevisiae has been well established, with only $5 \%$ of the $A$. gossypii annotated genes having no homologues in S. cerevisiae [3]. Despite the close phylogenetic relationship between these organisms, depicted in many works $[22,45,46]$, contrary to $S$. cerevisiae, A. gossypii is a pre-WGD organism, as $K$. lactis. Thus, the re-annotation performed in this work for A. gossypii was compared with that available in SGD (available for download in the section Curated Data) for $S$. cerevisiae and the one made by Dias et al. [14] for K. lactis.

\section{Enzymatic functions exclusively found in A. gossypii}

SGD is probably the best resource currently available for researchers working with $S$. cerevisiae, providing manually curated information. For an individual gene it is possible to consult a description of its function(s), from which, in many cases, an associated EC number may be 


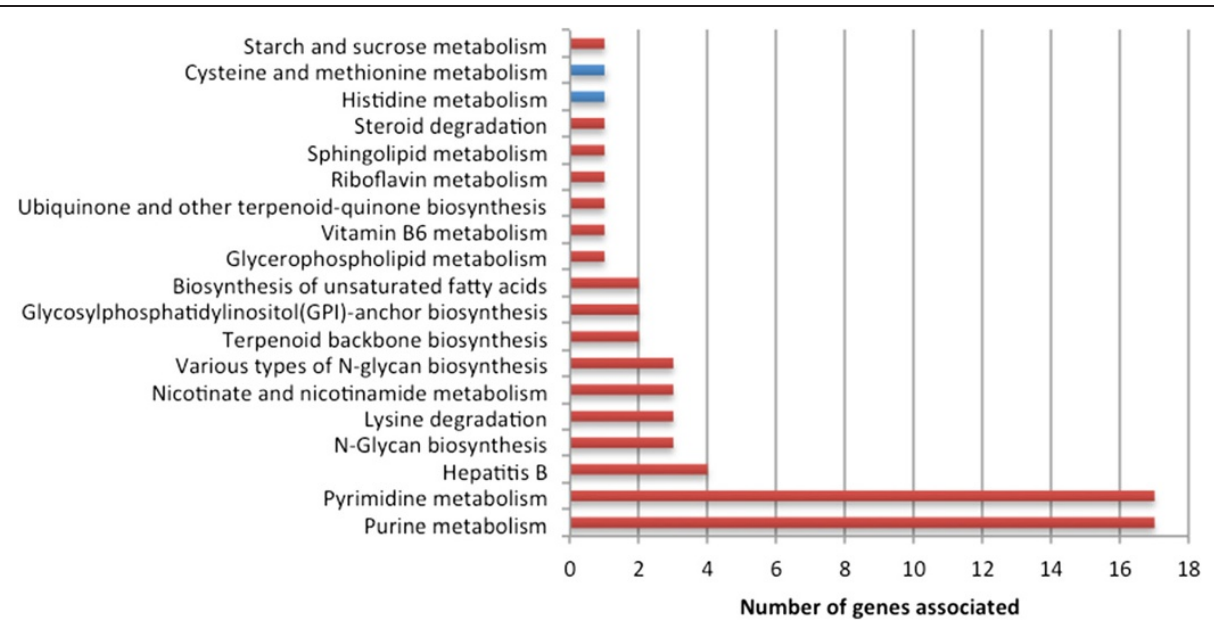

Figure 3 Distribution of genes exclusively annotated by KEGG over different metabolic pathways: blue bars represent metabolically relevant functions; red bars represent non-metabolically relevant functions.

retrieved. This platform also provides aggregated data of the overall genome (available for download), which was used on this work for comparison with the A. gossypii re-annotation. Given the discrepancies often found in both aggregated and non-aggregated data, both sources were used for comparison with our annotation. This comparison revealed only 13 exclusive EC numbers in our re-annotation. These exclusive EC numbers were associated to a wide range of metabolic pathways (Table 4), including the metabolism of different amino acids, lipids and sugars.

Our re-annotation was also compared with the one reported by Dias et al. [14] for K. lactis, with 90 enzymatic functions found exclusively in A. gossypii. Among the pathways with the highest number of exclusive enzymes associated (Figure 5), it was possible to find carbon source-related pathways, such as the starch and sucrose metabolism, amino acid-related pathways, such as those associated to phenylalanine, glycine, serine, threonine and tryptophan, several pathways related to lipids metabolism, the pathways of purine and pyrimidine metabolism, and also the pathway of riboflavin metabolism.

From the number of exclusive functions associated to each pathway it is not possible to predict the true extension of the possible metabolic differences existent between these species, as they strongly depend of the genomic context (total functions coded within the genome). However, significant differences between the metabolome of these species may be expected in some cases, as some exclusive enzymes are associated to important metabolic pathways (e.g. amino acids and lipids metabolism). In other cases, the presence of these exclusive functions may only mean

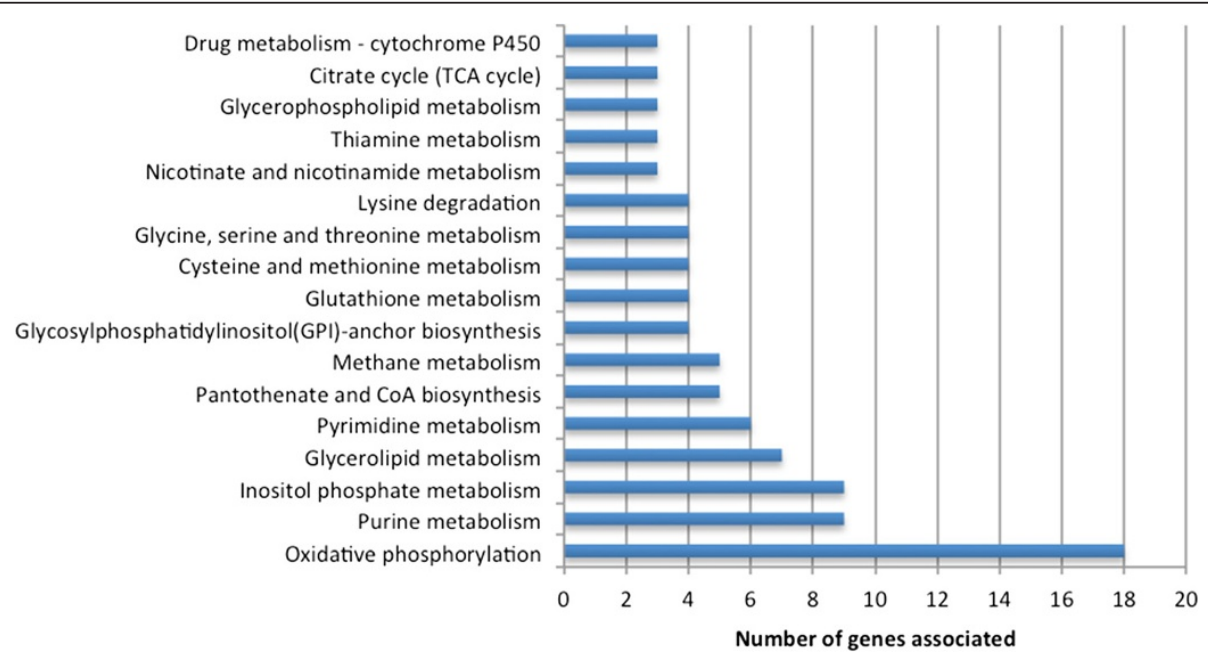

Figure 4 Distribution of the genes exclusively annotated by the current re-annotation over the metabolic pathways. Only the pathways with the highest number of associations are shown. 
Table 4 Enzymatic functions exclusively found in our re-annotation comparatively to S. cerevisiae (functions that were also found exclusively in our re-annotation comparatively to $K$. lactis are in italic; for each EC number the information source that was used to collect it is presented)

\begin{tabular}{|c|c|c|c|}
\hline EC number & Enzyme & Reaction(s) & Information source \\
\hline 1.13 .99 .1 & Inositol oxygenase & myo-inositol $+\mathrm{O}_{2}=$ D-glucuronate $+\mathrm{H}_{2} \mathrm{O}$ & Uniprot \\
\hline 1.14.11.1 & Gamma-butyrobetaine dioxygenase & $\begin{array}{l}\text { 4-trimethylammoniobutanoate }+ \text { 2-oxoglutarate }+ \\
\mathrm{O}_{2}=3 \text {-hydroxy-4-trimethylammoniobutanoate }+ \text { succinate }+\mathrm{CO}_{2}\end{array}$ & merlin \\
\hline 1.14.11.8 & Trimethyllysine dioxygenase & $\begin{array}{l}\mathrm{N} 6, \mathrm{~N} 6, \mathrm{~N} 6 \text {-trimethyl-L-lysine }+ \text { 2-oxoglutarate }+\mathrm{O}_{2}= \\
\text { 3-hydroxy-N6,N6,N6-trimethyl-L-lysine }+ \text { succinate }+\mathrm{CO}_{2}\end{array}$ & Uniprot \\
\hline 1.14.19.4 & $\Delta 8$-fatty-acid desaturase & $\begin{array}{l}\text { phytosphinganine }+ \text { reduced acceptor }+\mathrm{O}_{2}= \\
\text { Delta8-phytosphingenine }+ \text { acceptor }+2 \mathrm{H}_{2} \mathrm{O}\end{array}$ & merlin \\
\hline 1.14.19.6 & $\Delta 12$-fatty-acid desaturase & $\begin{array}{l}\text { acyl-CoA + reduced acceptor }+\mathrm{O}_{2}= \\
\text { Delta12-acyl-CoA }+ \text { acceptor }+2 \mathrm{H}_{2} \mathrm{O}\end{array}$ & merlin \\
\hline 1.4.3.21 & Primary-amine oxidase & $\mathrm{RCH}_{2} \mathrm{NH}_{2}+\mathrm{H}_{2} \mathrm{O}+\mathrm{O}_{2}=\mathrm{RCHO}+\mathrm{NH}_{3}+\mathrm{H}_{2} \mathrm{O}_{2}$ & Uniprot \\
\hline 2.1.1.79 & Cyclopropane synthetase & $\begin{array}{l}\text { S-adenosyl-L-methionine + phospholipid olefinic } \\
\text { fatty acid = S-adenosyl-L-homocysteine + } \\
\text { phospholipid cyclopropane fatty acid }\end{array}$ & merlin \\
\hline 2.4.1.80 & Ceramide glucosyltransferase & $\begin{array}{l}\text { UDP-glucose }+\mathrm{N} \text {-acylsphingosine }=\text { UDP }+ \\
\text { D-glucosyl-N-acylsphingosine }\end{array}$ & merlin \\
\hline 3.2.1.96 & Endo-beta-N-acetylglucosaminidase & $\begin{array}{l}\text { Endohydrolysis of the N,N'-diacetylchitobiosyl unit in } \\
\text { high-mannose glycopeptides and glycoproteins } \\
\text { containing the -[Man(GlcNAc)2]Asn- structure. }\end{array}$ & Uniprot \\
\hline 3.5.2.2 & Dihydropyrimidinase & 5,6-dihydrouracil $+\mathrm{H}_{2} \mathrm{O}=3$-ureidopropanoate & merlin \\
\hline 3.5.3.11 & Agmatinase & agmatine $+\mathrm{H}_{2} \mathrm{O}=$ putrescine + urea & AGD \\
\hline 3.5.99.6 & Glucosamine-6-phosphate deaminase & D-glucosamine 6-phosphate $+\mathrm{H}_{2} \mathrm{O}=$ D-fructose 6-phosphate $+\mathrm{NH}_{3}$ & AGD \\
\hline 4.6.1.13 & Phosphatidylinositol diacylglycerol-lyase & $\begin{array}{l}\text { 1-phosphatidyl-1D-myo-inositol = 1D-myo-inositol } \\
\text { 1,2-cyclic phosphate + 1,2-diacyl-sn-glycerol }\end{array}$ & merlin \\
\hline
\end{tabular}

an alternative reactive process. One example is 4- $\alpha$ glucanotransferase (2.4.1.25), exclusively found in $A$. gossypii when compared to $K$. lactis. According to KEGG, this enzyme only provides an alternative way to convert maltose into $\alpha$-D-glucose, which is achieved in $K$. lactis by alpha-glucosidase (3.2.1.20).

Among the enzymatic functions exclusively found in A. gossypii comparatively to both $S$. cerevisiae and K. lactis (italicized in Table 4), there is an Endo- $\beta$-N-acetylglucosaminidase (3.2.1.96), which is involved in the hydrolysis of the N,N'-diacetylchitobiosyl unit in high-mannose glycopeptides and glycoproteins containing the -[Man$\mathrm{GlcNAc}_{2}$ ]Asn- structure. According to KEGG's metabolic maps, this is the only route allowing the degradation of this type of glycan, therefore corresponding to an additional metabolic capacity comparatively to $S$. cerevisiae and $K$. lactis. This enzymatic activity has a broad presence reported in eukaryotes and some experimental evidences point to the existence of this activity in A. gossypii as well [6]. Consistently with our results, this enzymatic activity has not been detected in S. cerevisiae [47].

Another exclusive metabolic capacity in $A$. gossypii may be granted by a primary-amine oxidase (1.4.3.21), which allows the oxidation of a wide range of primary amines such as tyramine, phenethylamine or dopamine, thus influencing the metabolism of several amino acids (glycine, serine, threonine, tyrosine, phenylalanine). Another case corresponds to the presence of glucosamine6-phosphate deaminase (3.5.99.6), which enables the utilization of chitin as carbon source. Opposing its absence from $S$. cerevisiae [48] and $K$. lactis, the AER268W gene from A. gossypii has reported similarities in AGD to SPCC16C4.10 from Schizosaccharomyces pombe, which is associated to the referred enzyme. This phenotype was experimentally confirmed by a positive growth of $A$. gossypii on D-glucosamine (a product of chitin degradation) as sole carbon source (data not shown), which requires the presence of the referred enzyme.

Associated to lysine degradation, $\gamma$-butyrobetaine dioxygenase (1.14.11.1) was another enzymatic function also found exclusively in A. gossypii. $\gamma$-butyrobetaine dioxygenase and trimethyllysine dioxygenase (1.14.11.8) are directly involved in the hydrolysis of protein-lysine and the final production of carnitine, a capacity that seems to be absent in S. cerevisiae [49].

No exclusive enzymes were found directly associated to the riboflavin metabolism that could explain the $A$. gossypii over-producing capacity for this compound. This emphasizes that, although the presence of genes is important, their regulation is often determinant, as was previously known for riboflavin biosynthesis [50,51]. It should be reminded in this context that the simple 


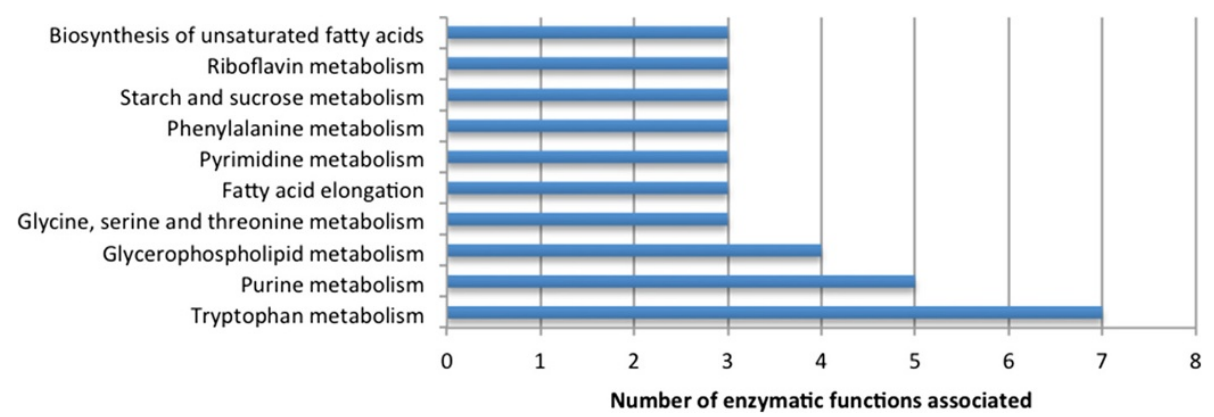

Figure 5 Distribution of the enzymes exclusively found in A. gossypii comparatively to K. lactis over different metabolic pathways.

presence of a gene with a given function does not mean the manifestation of the associated phenotype, which also reports to an important limitation of stoichiometric metabolic models. Also, a good example in this context is the work of Prinz et al. [44], who reported the role of regulatory mechanisms over the $26 \mathrm{~S}$ proteasome as a determinant of filamentous form-growth for a diploid budding-yeast.

When comparing only with $S$. cerevisiae, the exclusive presence of an inositol oxygenase (1.13.99.1) in A. gossypii may allow the production of glucuronic acid from myo-inositol, which is one of the few catabolic pathways for this compound. Beyond that, myo-inositol was reported as an important factor on riboflavin production through regulatory mechanisms [52]. Therefore, the presence of the referred enzyme could eventually influence the production of riboflavin. Also related to inositol metabolism is phosphatidylinositol diacylglycerol-lyase (4.6.1.13), involved on the hydrolysis of 1-phosphatidylD-myo-inositol to 1D-myo-inositol 1,2-cyclic phosphate and diacylglycerol. Like myo-inositol, diacylglycerol is a key element in several cellular signal transduction pathways [53]. Therefore, an alteration on its intracellular pool may lead to some differences on cellular processes associated to these mechanisms.

$\Delta 8$-fatty acid desaturase (1.14.19.4) and $\Delta 12$-fatty acid desaturase (1.14.19.6) play important roles on the production of unsaturated fatty acids. Their absence in $S$. cerevisiae therefore confirms $\Delta 9$-fatty acid desaturase as the only fatty acid desaturase in S. cerevisiae [54] and its incapacity to produce PUFAs (poly-unsatturated fatty acids) [55]. The presence of these three enzymes in $A$. gossypii, on the other hand, suggests that it may be able to produce this class of compounds, as was already reported by Stahmann et al. [56].

Dihydropyrimidinase (3.5.2.2), which was associated by merlin to the A. gossypii ACR027C gene, belongs to a strict group of enzymes associated to the reductive catabolism of pyrimidines, enabling the growth on uracil, dihydrouracil, beta-ureidopropionate or beta-alanine as sole nitrogen source. Its absence from $S$. cerevisiae was supported by Andersen [57], who verified the incapacity of $S$. cerevisiae to grow at any of the referred substrates. Additionally, the author suggested that the capacity to use uracil, dihydrouracil or beta-ureidopropionate as sole nitrogen sources might have been lost at the time of the yeast genome duplication. This could indicate that A. gossypii (pre-WGD) may indeed present this metabolic capacity, similarly to what the author observed for K. lactis (pre-WGD).

Equally important is the capacity to produce cerebroside, a common membrane lipid present in fungi, which is generated by the action of ceramide glucosyltransferase (2.4.1.80). Contrarily to several yeasts, such as Saccharomyces kluyveri, Zygosaccharomyces cidri, Zygosaccharomyces fermentati, K. lactis, Kluyveromyces thermotolerans or Kluyveromyces walti, S. cerevisiae lacks this enzyme [58]. The presence of this enzyme in A. gossypii can thus lead to some differences in the composition of the cell membrane comparatively to $S$. cerevisiae.

A more peculiar case corresponds to the exclusive presence of cyclopropane synthetase (2.1.1.79), which catalyzes the production of cyclopropane fatty acids (CFAs). This enzymatic function was associated to AFR735W using merlin (with a 68\% identity); however, no reports were found in the literature for the presence of this enzyme in any organism closely-related to $A$. gossypii, although being present in KEGG's annotation for Neurospora crassa.

Finally, the possible presence of agmatinase (3.5.3.11) activity in A. gossypii would represent a significant modification both on the catabolism of arginine and in the synthesis of polyamines as it provides an unusual route comparatively to what is known for the degradation of arginine [59]. Additionally, it also corresponds to a new path for the production of putrescine and subsequent synthesis of polyamine, acting as an alternative to ornithine decarboxylase (ODC), as was previously demonstrated by Klein et al. [60]. According to Coffino [61] this alternative pathway is however still not very clear in eukaryotes.

When comparing only to K. lactis, the tryptophan metabolism was clearly the pathway with the highest number of enzymes found exclusively in A. gossypii. It was 
verified that five of them are associated to the genes $B N A 1, B N A 2, B N A 4, B N A 5$ and BNA7, all involved in the kynurenine pathway for NAD biosynthesis. This result confirms the previous findings of $\mathrm{Li}$ and $\mathrm{Bao}$ [62], who verified the absence of this route in $K$. lactis, leading to an auxotrophy for nicotinic acid.

Within purine metabolism, five enzymes were found exclusively on A. gossypii covering, among others, the metabolism of GTP and glyoxylate, which have a reported connection to riboflavin metabolism. GTP is one of the precursors of riboflavin biosynthesis, while the glyoxylate cycle is directly involved in the formation of riboflavin precursors when plant oils are used as sole carbon sources [63].

Some differences were also found in the metabolism of different types of lipids, namely glycerophospholipids and unsaturated fatty acids. Within the synthesis of phosphatidylethanolamines (PE), it was verified that ethanolamine-phosphotransferase (2.7.8.1) and ethanolamine kinase (2.7.1.82) activities, both coded by EKI1, are absent in K. lactis. This result indicates that PE synthesis through the Kennedy pathway may not be possible on $K$. lactis, being exclusively dependent on the CDPDAG pathway [64]. Also, the capacity to utilize diacylglycerol (DAG) for the synthesis of phospholipids may be impaired, as the DGK1-coded diacylglycerol kinase (2.7.1.174) is equally absent in K. lactis, which would mean that cells would not be able to grow in a state where the de novo synthesis of fatty acids can not occur [64]. Relatively to the elongation of fatty acids and the synthesis of unsaturated fatty acids, three enzymes were also lacking in the re-annotation of Dias et al. [14], however, strong evidences were found for their presence in the K. lactis genome. Very-long-chain 3-oxoacyl-CoA reductase (1.1.1.330) was recently assigned by Swiss-Prot to KLLA0B09812g, while for the very-long-chain enoylCoA reductase (1.3.1.93) and enoyl-CoA hydratase (4.2.1.17) coding genes, results from pBLAST searches indicated possible homologues in this organism.

In what concerns the metabolism of amino acids, beyond tryptophan, some differences were also found for glycine, serine, threonine and phenylalanine, among others. Low-specificity L-threonine aldolase (4.1.2.48) was assigned exclusively to A. gossypii by homology to GLY1 from S. cerevisiae. Its absence from $K$. lactis may lead to slight differences in the riboflavin biosynthesis as it is directly involved in the formation of glycine, an important precursor in the de novo purine biosynthesis [65]. Since it is less specific than L-threonine aldolase (4.1.2.5), the presence of this enzyme in A. gossypii may cause a higher formation of glycine, as more substrate for this conversion is available. Associated to the metabolism of serine, D-serine hydrolase (4.3.1.18) was not annotated on $K$. lactis, which may indicate its incapacity to convert D-serine into pyruvate. This contradicts the results from a pBLAST search indicating KLLA0C11011g from $K$. lactis (74\% identity) as a putative homologue of the $S$. cerevisae FSH1, which could indicate a possible error in the re-annotation of this gene. A similar case was ARO10-coded phenylpyruvate decarboxylase (4.1.1.43), involved in the Ehrlich pathway of amino acids catabolism. Although absent from Dias et al. [14] re-annotation, pBLAST (e-value of 1E-30) indicated a possible homologue in that organism.

Regarding carbon metabolism, three enzymes were exclusively found in A. gossypii associated to the metabolism of starch and sucrose. Endopolygalacturonase (3.2.1.15) catalyzes de hydrolysis of (1->4)-alpha-D-galactosiduronic linkages in pectate and other galacturonans (information retrieved from KEGG), allowing the utilization of pectin as nutrient. This result contradicts the previous observations of Murad and Foda [66], who reported the production of this enzyme on $K$. lactis when grown on a pectin-based substrate. The other two enzymes were 4alpha-glucanotransferase (2.4.1.25) and amylo-alpha-1,6glucosidase (3.2.1.33), which constitute the glycogen debranching system (GDB1), essential for glycogen utilization as carbon source [67]. This result suggests an inability of $K$. lactis to use this substrate, or an alternative route to make it, which represents a significant metabolic trait.

As can be seen on Figure 5, three enzymes were found within the pathway of riboflavin biosynthesis as present in A. gossypii and not present in $K$. lactis. Flavin reductase (1.5.1.30), associated to the bifunctional $A R O 2$ gene from $S$. cerevisiae, is involved in the reduction of FAD to $\mathrm{FADH}_{2}$, the first one being one of the products from riboflavin degradation. One other enzyme, 2,5-diamino6-(ribosylamino)-4(3H)-pyrimidinone 5'-phosphate reductase (1.1.1.302), catalyzes the second step of riboflavin biosynthesis and is critical for this route. Although not referred by Dias et al. [14], this enzyme was already reported in $K$. lactis [68] and was recently assigned by Swiss-Prot to the K. lactis KLLA0F21120g gene.

\section{Enzymatic functions exclusively found in other organisms}

There were some biological functions absent in A. gossypii (Figure 6) that work as differentiating factors from the other two organisms.

From Figure 6 it is possible to verify that the distribution of the enzymatic functions exclusively found in $S$. cerevisiae and $K$. lactis comparatively to $A$. gossypii was quite similar, with several pathways found in common.

Similarly to what was observed for the enzymatic functions exclusively found in A. gossypii (Figure 5), the metabolism of purines and pyrimidines was also one of the pathways with more associated enzymatic functions absent in A. gossypii. The oxidation of 5-hydroxyisourate to (S)-allantoin, mediated by uric acid oxidase (1.7.3.3) 


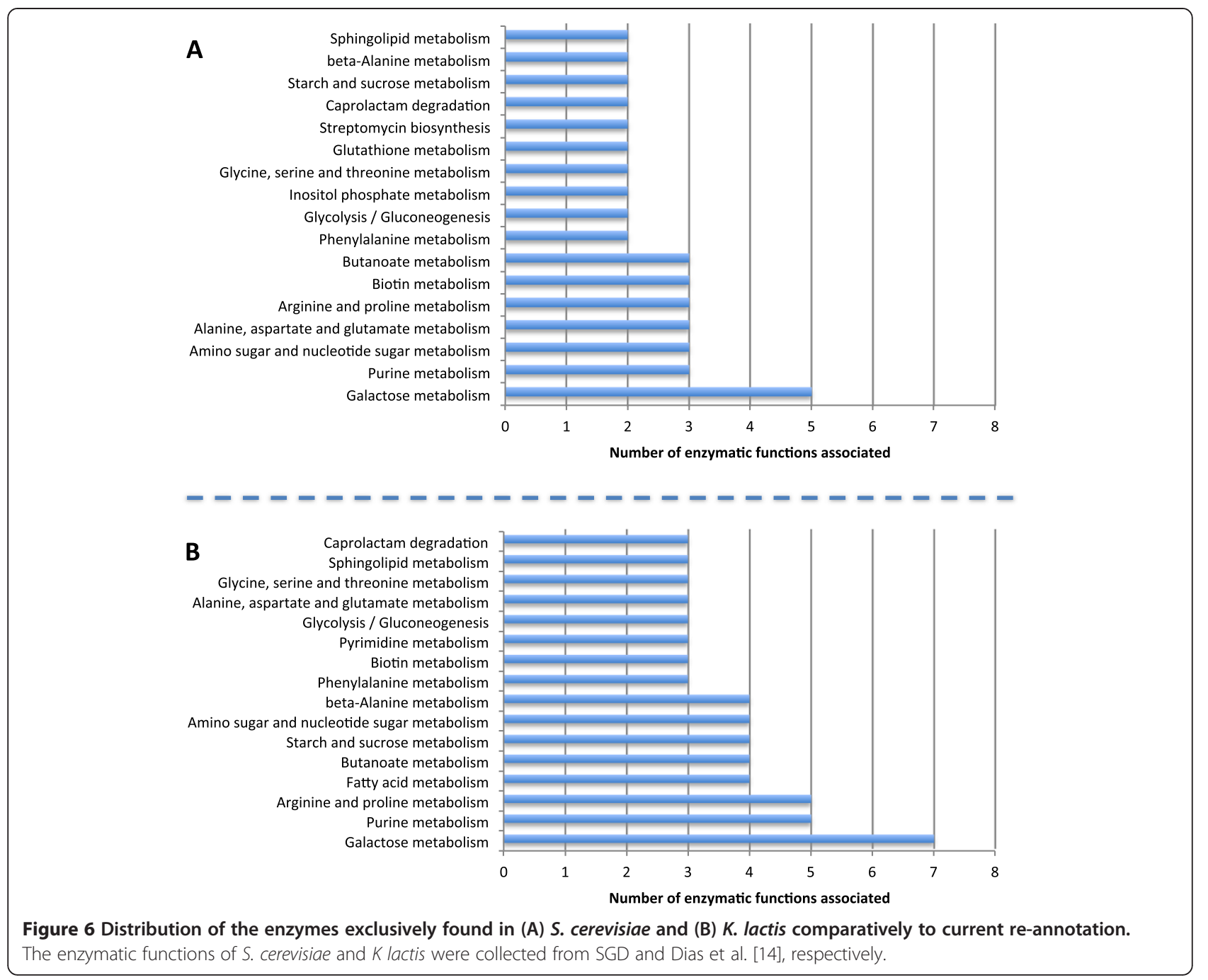

and hydroxyisourate hydrolase (3.5.2.17), was one of the functions involved in these pathways that was exclusively found on K. lactis. A similar case was pseudouridine kinase (2.7.1.83), which has been reported for several fungus, but is absent in S. cerevisiae and A. gossypii [69]. This particular function may allow complementing a deficiency on uracil production using pseudouridine, as reported by Preumont et al. [69] for E. coli. Associated to the deamination of purines, $A A H 1$-coded adenine deaminase (3.5.4.2) and GUD1-coded guanine deaminase (3.5.4.3) were not found in A. gossypii, impairing the formation of hypoxanthine and xanthine from adenine and guanine, respectively, and ultimately affecting the purine salvage pathway. Saint-Marc et al. [70] reported that a S. cerevisiae $\Delta a a h 1$ strain showed a slight growth defect in the presence of adenine. According to the authors, in the presence of adenine and in the absence of Amd1p, the synthesis of IMP and GMP would take place mostly through an AAH1, HPT1-mediated route. The most unexpected finding corresponded, however, to the exclusive presence of $\beta$-alanine synthase (3.5.1.6) on $K$. lactis, which is directly involved in the catabolism of pyrimidines. According to LaRue and Spencer [71], most of the fungi do not present this specific route, impairing the utilization of pyrimidines (or their degradation products) as sole nitrogen sources. The KLLA0D03520g gene from $K$. lactis showed, however, a significant similarity to PYD3 from S. kluyveri, which codes for a $\beta$-alanine synthase, indicating that this capability may be present on this organism as well. A final difference in the de novo biosynthesis of pyrimidines consists on the URA1-coded dihydroorotate dehydrogenase (DHOD) (1.3.98.1), involved in the de novo biosynthesis of pyrimidines, which is absent in A. gossypii. As described by Hall et al. [72], this seems to be an example of horizontal gene transfer from bacterial lineages, and more specifically, a case of gene displacement. URA1 codes for a DHOD type 1, which is optimized for anaerobic conditions. According to Hall et al. [72], the fixation of this bacterial gene in the genome of S. cerevisiae may indicate that its evolution involved adaptation to 
anaerobic environments, providing the selective pressure to maintain the referred gene. On the other hand, URA9, which has a homologue in A. gossypii but not in S. cerevisiae, codes for a type 2 DHOD (1.3.5.2) and seems to be oriented for aerobic conditions, corroborating the difficulty of $A$. gossypii to grow in anaerobic conditions [72].

In terms of carbon metabolism the main differences were found associated to the utilization of lactose and galactose. In contrast to $S$. cerevisiae and $K$. lactis, four enzymes of the Leloir pathway [73] were not found in $A$. gossypii, namely the GAL1-coded galactokinase (2.7.1.6), GAL7-coded galactose 1-phosphate uridyltransferase (2.7.7.12), GAL10-coded UDP-galactose 4-epimerase (5.1.3.2) and GAL10-coded aldose 1-epimerase (5.1.3.3). This result supports the inability reported for $A$. gossypii to grow on galactose [74], as these are critical enzymes on this metabolic route. Also, $\beta$-galactosidase (3.2.1.23), which is associated to lactose utilization, was only found in $K$. lactis, confirming the exclusive presence of this important trait on this organism. $\beta$-glucosidase (3.2.1.21) was another exclusive enzyme found on this organism. This enzyme, which catalyzes the conversion of cellobiose to $\beta$-Dglucose, is directly involved in the degradation of cellulose [75], assuming a considerable importance in a biotechnological context. Both A. gossypii and S. cerevisiae present genes coding for the glucan 1,3- $\beta$-glucosidase (3.2.1.58); however, this enzyme acts on $\beta-(1->3)$ bonds, impairing therefore cellobiose degradation. A more interesting case refers to the utilization of maltose. Although isomaltase (3.2.1.10; coded by IMA1, IMA2, IMA3, IMA4 and IMA5) and maltase (3.2.1.20; coded by MAL12 and MAL32) were absent in $A$. gossypii, some old reports suggest the capability to utilize this carbon source [76]. More specifically, Mickelson [77] reported that A. gossypii was able to oxidize maltose, though more slowly comparatively to glucose and sucrose. Although no homologues were detected regarding the above-mentioned maltase and isomaltase, this phenotype can have occurred through other unknown systems allowing maltose utilization, such as 4-alpha-glucanotransferase (2.4.1.25). Another possibility that should be considered is the existence of small differences between different strains of the same specie as recently exposed by Ribeiro et al. [78] and by Dietrich et al. [79].

Within nitrogen metabolism, several differences were observed over different amino acids. Specifically associated to glutamate degradation, it was observed that GDH1 (or GDH3)-coded $\mathrm{NADP}^{+}$-dependent glutamate dehydrogenase was absent comparatively both with $S$. cerevisae and $K$. lactis, indicating that $A$. gossypii possibly does not have the glutamate dehydrogenase ammonium assimilation route [80]. Also, GAD1-coded glutamate decarboxylase was not present, impairing the gamma-aminobutyrate (GABA) route of glutamate degradation [81]. Involved in both alanine and glycine metabolism, AGX1-coded alanine:glyoxylate aminotransferase (2.6.1.44) was lacking on this organism, which may have a significant impact on riboflavin biosynthesis as it conducts one of the main routes for glycine formation on yeasts. This result confirms previous studies aiming the improvement of riboflavin production through the introduction of this route [82]. GCV1-coded T subunit of mitochondrial glycine decarboxylase complex was also not found in A. gossypii, which may suggest its incapacity to utilize glycine as sole nitrogen source [83]. Indirectly related to glycine synthesis, it was observed the exclusive presence of 2-hydroxyglutarate dehydrogenase (1.1.99.2) on $K$. lactis, which mediates the formation of 2-hydroxyglutarate from 2-oxoglutarate. Despite this absence, Albers et al. [84] have previously suggested that this activity may be present in $S$. cerevisiae associated to YERO81W and YILO74C, which have a homologue in A. gossypii (ACLO32C). Involved in the Ehrlich pathway of amino acids degradation, the absence of ARO9-coded tryptophan:phenylpyruvate transaminase (2.6.1.28) was verified in A. gossypii, confirming previous reports indicating its inability to degrade amino acids into fusel acids/fusel alcohols [85]. Relatively to sulfur-related amino acids, it was observed the lack of SPE4-coded spermine synthase (2.5.1.22), which is directly associated to spermine biosynthesis from $\mathrm{S}$-adenosylmethionine and the final production of volatile sulfur compounds (VSC). This result is in accordance with the previous study of Hébert et al. [86], who reported several differences in the sulfur amino acid pathways among hemiascomycetous yeast, such as the absence of SPE4 in A. gossypii.

Sphingolipid metabolism also presented a few differences among the species. The first one refers to DPL1encoded sphinganine-1-phosphate lyase (4.1.2.27), which was only found in S. cerevisiae and K. lactis. According to different reports $[87,88]$, this gene may play a critical role in the cell response to nutrient starvation through changes on the internal concentration of sphingosine-1-phosphate. More specifically, Gottlieb et al. [87] observed that a $\Delta d p l 1$ strain of $S$. cerevisiae presented an unusual accumulation of phosphorylated sphingoid bases, leading to an unregulated proliferation close to the stationary phase. The other difference is related to the exclusive presence of BDS1-encoded arylsulfatase (3.1.6.1) in S. cerevisiae and $K$. lactis. Similarly to the above mentioned URA1, this may also represent a case of horizontal gene transfer [72], which could explain its absence from $A$. gossypii. Like $U R A 1$, the acquisition of this function may have derived from an environmental pressure to utilize alternative sulfur sources, which may be beneficial in a constrained environment. Hall et al. [72] observed a small increase on the cell growth of wild-type $S$. cerevisiae comparatively to a $\Delta b d s 1$ strain when a medium supplemented with 4nitrocatechol sulfate was used. 
Still related to the overall metabolism of lipids, the $\beta$ oxidation of fatty acids also presented some differences. Contrarily to $K$. lactis, S. cerevisiae and A. gossypii did not present the set of enzymes constituted by 1.3.8.7, 1.3.8.8 and 1.3.8.9, which is directly involved in the first step of fatty acids $\beta$-oxidation. Instead, they presented an equivalent system associated to $P O X 1$ gene from $S$. cerevisiae and with a homologue in A. gossypii. The main difference between these systems lies on the type of electron transfer mechanism and intermediates. While the first system, mainly mitochondrial, uses an electron transport chain until the final receptor-oxygen-, the second one (POX1), which is peroxisomal, transfers instead the electrons directly to oxygen [89]. It is worth noting, however, that the presence of the first system in $K$. lactis is an unexpected finding, as fungal organisms are mainly characterized by the peroxisomal system [90].

A common reported phenotype in A. gossypii is its incapacity to synthesize biotin [91], confirmed on this case by the absence of $\mathrm{BIO} 2, \mathrm{BIO} 3$ and $\mathrm{BIO} 4$ coded enzymes, directly associated to this process [92], which were found in both $S$. cerevisiae and $K$. lactis. A similar result was found for myo-inositol, supported by the absence of INO1-coded myo-inositol-1-phosphate synthase (5.5.1.4). Also related to inositol biosynthesis, inositol-phosphate phosphatase (3.1.3.25) was exclusively found in S. cerevisiae (INM1 and INM2). However, contrarily to the previous case, this function seems not to be essential, as a double mutation of INM1 and INM2 did not affect growth and inositol biosynthesis in S. cerevisiae [93].

\section{Conclusions}

A functional re-annotation pipeline was designed and applied to the entire genome of A. gossypii rendering the first enzymatic functional annotation for this organism. After applying this pipeline, 847 genes were manually assigned with metabolic enzymatic functions. Additionally, the first annotation of the membrane transport proteins for this organism was also performed, which allowed to identify and classify 265 genes as potential membrane transporters. Comparatively to KEGG, this re-annotation allowed correcting the information of 95 genes of $A$. gossypii, ranging from completing EC numbers, adding new EC numbers or removing outdated information. Among these 95 genes it was possible to assign 22 new enzymatic functions that were completely absent from KEGG's annotation for $A$. gossypii, which corresponds to a significant improvement in the knowledge of its metabolism.

Also, a comparative analysis between the set of EC numbers associated to $S$. cerevisiae, K. lactis and A. gossypii provided an overall perspective over some of the main differences among their metabolism. When compared to $S$. cerevisae, A. gossypii only presented 13 exclusive EC numbers. This number was considerably higher when this comparison was made with $K$. lactis (90). In both cases these EC numbers were spread into different parts of the metabolism, namely carbon and nitrogen metabolism, lipids, purine and pyrimidines, among others, with no enriched part in particular. The similar happened for the enzymatic functions that were absent in $A$. gossypii but not in $S$. cerevisiae and $K$. lactis. These last analyses found fundamental evidences for some of the well-known phenotypes of $A$. gossypii such as the inability to use lactose and galactose, and to synthesize biotin and myo-inositol, among others.

This work reports the first manual genome-wide enzymatic functional annotation for the metabolism of $A$. gossypii. As manually curated data, it represents a high quality information source, providing an increased knowledge of the A. gossypii's metabolism. Additionally, the comparative study performed with the metabolism of the closelyrelated yeasts $S$. cerevisiae and $K$. lactis also provided relevant insights into the $A$. gossypii's metabolism. Together with the membrane transporters annotation, this whole data set constitutes a solid and more complete platform for the development of different studies, namely in the field of fungal biology and systems biology.

\section{Availability of supporting data}

The data sets supporting the results of this article are included within the article (and its additional files).

\section{Additional files}

Additional file 1: Figure A1- Pipeline used for the metabolic functional re-annotation of the $A$. gossypii genome; Detailed description of the pipeline.

Additional file 2: Supplement 1-Metabolic genome-wide functional re-annotation of Ashbya gossypii ATCC 10895; Supplement 2- Overall statistics of the functional re-annotation; Supplement 3-Comparison of the annotations for the 668 genes annotated both on KEGG and on the current re-annotation; Supplement 4- Membrane transport proteins annotation of Ashbya gossypii ATCC 10895; Supplement 5- Overall statistics of the transport proteins annotation; Supplement 6- Set of EC numbers of S. cerevisiae (SGD), K. lactis (Dias et al. [14]) and $A$. gossypii (current re-annotation); Supplement 7- Genes annotated exclusively by KEGG and their classification into metabolic pathways; Supplement 8- Genes annotated exclusively in the current re-annotation and their classification into metabolic pathways; Supplement 9- Enzymatic functions exclusively found in A. gossypii comparatively to $S$. cerevisiae; Supplement 10- Enzymatic functions exclusively found in A. gossypii comparatively to K. lactis; Supplement 11- Enzymatic functions exclusively found in $S$. cerevisiae comparatively to $A$. gossypii; Supplement 12- Enzymatic functions exclusively found in $K$. lactis comparatively to $A$. gossypii.

\section{Abbreviations}

AGD: Ashbya Genome Database; BLAST: Basic local alignment search tool; CFAs: Cyclopropane fatty acids; DAG: Diacylglycerol; DHOD: Dihydroorotate dehydrogenase; EC: Enzyme commission; GABA: Gamma-aminobutyrate; GO: Gene Ontology; GSMMs: Genome-scale metabolic models;

HMMs: Hidden markov models; KEGG: Kyoto encyclopedia of genes and genomes; ODC: Ornithine decarboxylase; PE: Phosphatidylethanolamines; PUFAs: Poly-unsatturated fatty acids; SGD: Saccharomyces Genome Database; 
SW: Smith-Waterman; TC: Transporter classification; TCDB: Transporter classification database; TCGs: Transporter candidate genes; TMHMM: TransMembrane prediction using Hidden Markov Models; VSC: Volatile sulfur compounds; WGD: Whole genome duplication.

\section{Competing interests}

The authors declare that they have no competing interests.

\section{Authors' contributions}

DG performed the annotation and the metabolic comparative analysis, and elaborated the manuscript. TQA participated in the metabolic comparative analysis and in the manuscript elaboration. OD participated in the annotation and in the manuscript elaboration. ECF participated in the design of the study and helped to draft the manuscript. LD and IR conceived the study, participated in its design and coordination and helped to draft the manuscript. All authors read and approved the final manuscript.

\section{Acknowledgements and funding}

Research described in this article was financially supported by FEDER and "Fundação para a Ciência e a Tecnologia" (FCT): Project AshByofactory (PTDC/EBB-EBI/101985/2008 - FCOMP-01-0124-FEDER-009701), Strategic Project PEst-OE/EQB/LA0023/2013, Project "Biolnd - Biotechnology and Bioengineering for improved Industrial and Agro-Food processes, REF. NORTE-07-0124-FEDER-000028" Co-funded by the Programa Operacional Regional do Norte (ON.2 - O Novo Norte), QREN, FEDER and the PhD grant to DG (SFRH/BD/88623/2012).

Received: 30 April 2014 Accepted: 15 August 2014

Published: 24 September 2014

\section{References}

1. Wendland J, Walther A: Ashbya gossypii: a model for fungal developmental biology. Nat Rev Microbiol 2005, 3:421-429.

2. Schmitz HP, Philippsen P: Evolution of multinucleated Ashbya gossypii hyphae from a budding yeast-like ancestor. Fungal Biol 2011, 115:557-568.

3. Dietrich FS, Voegeli S, Brachat S, Lerch A, Gates K, Steiner S, Mohr C, Pohlmann R, Luedi P, Choi S, Wing RA, Flavier A, Gaffney TD, Philippsen P: The Ashbya gossypii genome as a tool for mapping the ancient Saccharomyces cerevisiae genome. Science 2004, 304:304-307.

4. Ribeiro O, Wiebe M, IImen M, Domingues L, Penttila M: Expression of Trichoderma reesei cellulases CBHI and EGI in Ashbya gossypii. Appl Microbiol Biotechnol 2010, 87:1437-1446.

5. Magalhães F, Aguiar TQ, Oliveira CM, Domingues L: High-level expression of Aspergillus niger $\beta$-galactosidase in Ashbya gossypii. Biotechnol Prog 2014, 30:261-268.

6. Aguiar TQ, Maaheimo H, Heiskanen A, Wiebe MG, Penttilä M, Domingues L: Characterization of the Ashbya gossypii secreted N-glycome and genomic insights into its N-glycosylation pathway. Carbohydr Res 2013, 381:19-27.

7. Porro D, Sauer M, Branduardi P, Mattanovich D: Recombinant protein production in yeasts. Mol Biotechnol 2005, 31:245-259.

8. Demain AL, Vaishnav P: Production of recombinant proteins by microbes and higher organisms. Biotechnol Adv 2009, 27:297-306.

9. Baart GJ, Martens DE: Genome-scale metabolic models: reconstruction and analysis. Methods Mol Biol 2012, 799:107-126.

10. Rocha I, Forster J, Nielsen J: Design and application of genome-scale reconstructed metabolic models. Methods Mol Biol 2008, 416:409-431.

11. National Center for Biotechnology Information. [http://www.ncbi.nlm.nih. gov/]

12. Cherry JM, Hong EL, Amundsen C, Balakrishnan R, Binkley G, Chan ET, Christie KR, Costanzo MC, Dwight SS, Engel SR, Fisk DG, Hirschman JE, Hitz BC, Karra K, Krieger CJ, Miyasato SR, Nash RS, Park J, Skrzypek MS, Simison M, Weng S, Wong ED: Saccharomyces Genome Database: the genomics resource of budding yeast. Nucleic Acids Res 2012, 40:D700-D705.

13. Gattiker A, Rischatsch R, Demougin P, Voegeli S, Dietrich FS, Philippsen P, Primig M: Ashbya Genome Database 3.0: a cross-species genome and transcriptome browser for yeast biologists. BMC Genomics 2007, 8:9.

14. Dias O, Gombert AK, Ferreira EC, Rocha I: Genome-wide metabolic (re-) annotation of Kluyveromyces lactis. BMC Genomics 2012, 13:517.

15. Ouzounis CA, Karp PD: The past, present and future of genome-wide re-annotation. Genome Biol 2002, 3:1-6.
16. Gundogdu O, Bentley SD, Holden MT, Parkhill J, Dorrell N, Wren BW: Re-annotation and re-analysis of the Campylobacter jejuni NCTC11168 genome sequence. BMC Genomics 2007, 8:162.

17. Camus JC, Pryor MJ, Medigue C, Cole ST: Re-annotation of the genome sequence of Mycobacterium tuberculosis H37Rv. Microbiology 2002, 148:2967-2973.

18. Riley M, Abe T, Arnaud MB, Berlyn MK, Blattner FR, Chaudhuri RR, Glasner JD, Horiuchi T, Keseler IM, Kosuge T, Mori H, Perna NT, Plunkett G 3rd, Rudd KE, Serres $\mathrm{MH}$, Thomas GH, Thomson NR, Wishart D, Wanner BL: Escherichia coli K-12: a cooperatively developed annotation snapshot-2005. Nucleic Acids Res 2006, 34:1-9

19. Barbe V, Cruveiller S, Kunst F, Lenoble P, Meurice G, Sekowska A, Vallenet D, Wang T, Moszer I, Médigue C, Danchin A: From a consortium sequence to a unified sequence: the Bacillus subtilis 168 reference genome a decade later. Microbiology 2009, 155:1758-1775.

20. Haas BJ, Wortman JR, Ronning CM, Hannick LI, Smith RK, Maiti R, Chan AP, Yu C, Farzad M, Wu D, White O, Town CD: Complete reannotation of the Arabidopsis genome: methods, tools, protocols and the final release. BMC Biol 2005, 3:7.

21. Dias O, Rocha M, Ferreira EC, Rocha I: Merlin: Metabolic models reconstruction using genome-scale information. In Proceedings of the 11th International Symposium on Computer Applications in Biotechnology (CAB 2010). Edited by Banga JR, Bogaerts $P$, Van Impe JFM, Dochain D, Smets I. Leuven, Belgium: Oude Valk College; 2010:120-125.

22. Kurtzman $C P$, Robnett $C J$ : Phylogenetic relationships among yeasts of the 'Saccharomyces complex' determined from multigene sequence analyses. FEMS Yeast Res 2003, 3:417-432

23. UniProt. [http://www.uniprot.org/]

24. Consortium UP: The Universal Protein Resource (UniProt). Nucleic Acids Res 2007, 35:D193-D197.

25. Saccharomyces Genome Database. [http://www.yeastgenome.org/]

26. Ashbya Genome Database. [http://agd.vital-it.ch/index.html]

27. Transporter Classification Database. [http://www.tcdb.org/]

28. Saier MH, Tran CV, Barabote RD: TCDB: the Transporter Classification Database for membrane transport protein analyses and information. Nucleic Acids Res 2006, 34:D181-D186.

29. Kyoto Encyclopedia of Genes and Genomes. [http://www.genome.jp/ kegg/]

30. Kanehisa M, Goto S: KEGG: Kyoto Encyclopedia of Genes and Genomes. Nucleic Acids Res 2000, 28:27-30.

31. BRENDA. [http://www.brenda-enzymes.info/]

32. Schomburg I, Chang A, Schomburg D: BRENDA, enzyme data and metabolic information. Nucleic Acids Res 2002, 30:47-49.

33. PEDANT 3 database. [http://pedant.gsf.de/]

34. Walter MC, Rattei T, Arnold R, Güldener U, Münsterkötter M, Nenova K, Kastenmüller G, Tischler P, Wölling A, Volz A, Pongratz N, Jost R, Mewes HW, Frishman D: PEDANT covers all complete RefSeq genomes. Nucleic Acids Res 2009, 37:D408-D411.

35. Altschul SF, Gish W, Miller W, Myers EW, Lipman DJ: Basic local alignment search tool. J Mol Biol 1990, 215:403-410.

36. Eddy SR: Profile hidden Markov models. Bioinformatics 1998, 14:755-763.

37. Dias O: Reconstruction of the Genome-scale Metabolic Network of Kluyveromyces lactis. PhD thesis. University of Minho, School of Engineering; 2013.

38. TMHMM Server v.2.0. [http://www.cbs.dtu.dk/services/TMHMM-2.0/]

39. Smith TF, Waterman MS: Identification of common molecular subsequences. J Mol Biol 1981, 147:195-197.

40. Ruepp A, Zollner A, Maier D, Albermann K, Hani J, Mokrejs M, Tetko I, Güldener U, Mannhaupt G, Münsterkötter M, Mewes HW: The FunCat, a functional annotation scheme for systematic classification of proteins from whole genomes. Nucleic Acids Res 2004, 18:5539-5545.

41. Brachat S, Dietrich F, Voegeli S, Gaffney T, Philippsen P: The genome of the filamentous fungus Ashbya gossypii: annotation and evolutionary implications. Comp Genomics-Topics Curr Genet 2006, 15:197-232.

42. Resende T, Correia DM, Rocha M, Rocha I: Re-annotation of the genome sequence of Helicobacter pylori 26695. J Integr Bioinform 2013, 10:1-13.

43. Thiele I, Palsson $B \varnothing$ : A protocol for generating a high-quality genome-scale metabolic reconstruction. Nat Prot 2010, 5:93-121.

44. Prinz S, Avila-Campillo I, Aldridge C, Srinivasan A, Dimitrov K, Siegel AF, Galitski T: Control of yeast filamentous-form growth by modules in an integrated molecular network. Genome Res 2004, 14:380-390. 
45. Fitzpatrick DA, Logue ME, Stajich JE, Butler G: A fungal phylogeny based on 42 complete genomes derived from supertree and combined gene analysis. BMC Evol Biol 2006, 6:99

46. Wang $H, X u Z$, Gao L, Hao B: A fungal phylogeny based on 82 complete genomes using the composition vector method. BMC Evol Biol 2009, 9:195.

47. Suzuki T, Yano K, Sugimoto S, Kitajima K, Lennarz WJ, Inoue S, Inoue Y, Emori $Y$ : Endo-beta-N-acetylglucosaminidase, an enzyme involved in processing of free oligosaccharides in the cytosol. Proc Natl Acad Sci U S A 2002, 99:9691-9696.

48. Wendland J, Schaub Y, Walther A: N-acetylglucosamine utilization by Saccharomyces cerevisiae based on expression of Candida albicans NAG genes. Appl Environ Microbiol 2009, 75:5840-5845.

49. Strijbis K, van Roermund CW, Hardy GP, van den Burg J, Bloem K, de Haan J, van Vlies N, Wanders RJ, Vaz FM, Distel B: Identification and characterization of a complete carnitine biosynthesis pathway in Candida albicans. FASEB J 2009, 23:2349-2359.

50. Schlösser T, Wiesenburg A, Gätgens C, Funke A, Viets U, Vijayalakshmi S, Nieland S, Stahmann KP: Growth stress triggers riboflavin overproduction in Ashbya gossypii. Appl Microbiol Biotechnol 2007, 76:569-578.

51. Walther A, Wendland J: Yap1-dependent oxidative stress response provides a link to riboflavin production in Ashbya gossypii. Fungal Genet Biol 2012, 49:697-707.

52. Sengupta S, Kiruthiga S, Chandra TS: Antagonistic effect of myo-inositol on riboflavin production in two riboflavinogenic fungi Ashbya gossypii and Eremothecium ashbyii. Mycoscience 2013, 54:429-432.

53. Cerbón J, Falcon A, Hernández-Luna C, Segura-Cobos D: Inositol phosphoceramide synthase is a regulator of intracellular levels of diacylglycerol and ceramide during the G1 to $\mathrm{S}$ transition in Saccharomyces cerevisiae. Biochem J 2005, 388:169-176.

54. Stukey JE, McDonough VM, Martin CE: Isolation and characterization of OLE1, a gene affecting fatty acid desaturation from Saccharomyces cerevisiae. J Biol Chem 1989, 264:16537-16544.

55. Watanabe K, Oura T, Sakai H, Kajiwara S: Yeast $\Delta 12$ Fatty Acid Desaturase: Gene Cloning, Expression, and Function. Biosci Biotechnol Biochem 2004, 68:721-727.

56. Stahmann KP, Kupp C, Feldmann SD, Sahm H: Formation and degradation of lipid bodies found in the riboflavin-producing fungus Ashbya gossypii. Appl Microbiol Biotechnol 1994, 42:121-127.

57. Andersen G: Uracil and beta-alanine degradation in Saccharomyces kluyveri - Discovery of a novel catabolic pathway. PhD thesis. Technical University of Denmark; 2006.

58. Takakuwa N, Kinoshita M, Oda Y, Ohnishi M: Existence of cerebroside in Saccharomyces kluyveri and its related species. FEMS Yeast Res 2002 2:533-538.

59. Brandriss MC, Magasanik B: Proline: an essential intermediate in arginine degradation in Saccharomyces cerevisiae. J Bacteriol 1980, 143:1403-1410.

60. Klein RD, Geary TG, Gibson AS, Favreau MA, Winterrowd CA, Upton SJ, Keithly JS, Zhu G, Malmberg RL, Martinez MP, Yarlett N: Reconstitution of a bacterial/plant polyamine biosynthesis pathway in Saccharomyces cerevisiae. Microbiology 1999, 145:301-307.

61. Coffino P: Regulation of cellular polyamines by antizyme. Nat Rev Mol Cell Biol 2001, 2:188-194.

62. Li YF, Bao WG: Why do some yeast species require niacin for growth? Different modes of NAD synthesis. FEMS Yeast Res 2007, 7:657-664.

63. Schmidt G, Stahmann KP, Kaesler B, Sahm H: Correlation of isocitrate lyase activity and riboflavin formation in the riboflavin overproducer Ashbya gossypii. Microbiology 1996, 142:419-426.

64. Henry SA, Kohlwein SD, Carman GM: Metabolism and regulation of glycerolipids in the yeast Saccharomyces cerevisiae. Genetics 2012 190:317-349.

65. Monschau N, Sahm H, Stahmann KP: Threonine aldolase overexpression plus threonine supplementation enhanced riboflavin production in Ashbya gossypii. Appl Environ Microbiol 1998, 64:4283-4290.

66. Murad HA, Foda MS: Production of yeast polygalacturonase on dairy wastes. Bioresour Technol 1992, 41:247-250.

67. Teste MA, Enjalbert B, Parrou JL, François JM: The Saccharomyces cerevisiae YPR184w gene encodes the glycogen debranching enzyme. FEMS Microbiol Lett 2000, 193:105-110.

68. Voronovsky AY, Abbas CA, Dmytruk KV, Ishchuk OP, Kshanovska BV, Sybirna KA, Gaillardin C, Sibirny AA: Candida famata (Debaryomyces hansenii)
DNA sequences containing genes involved in riboflavin synthesis Yeast 2004, 21:1307-1316.

69. Preumont A, Snoussi K, Stroobant V, Collet JF, Van Schaftingen E: Molecular identification of pseudouridine-metabolizing enzymes. J Biol Chem 2008, 283:25238-25246.

70. Saint-Marc C, Pinson B, Coulpier F, Jourdren L, Lisova O, Daignan-Fornier B: Phenotypic consequences of purine nucleotide imbalance in Saccharomyces cerevisiae. Genetics 2009, 183:529-538.

71. LaRue TA, Spencer JFT: The utilization of purines and pyrimidines by yeasts. Can J Microbiol 1968, 14:79-86.

72. Hall C, Brachat S, Dietrich FS: Contribution of horizontal gene transfer to the evolution of Saccharomyces cerevisiae. Eukaryot Cell 2005, 4:1102-1115.

73. Holden HM, Rayment I, Thoden JB: Structure and function of enzymes of the Leloir pathway for galactose metabolism. J Biol Chem 2003, 278:43885-43888.

74. Hittinger CT, Rokas A, Carroll SB: Parallel inactivation of multiple GAL pathway genes and ecological diversification in yeasts. Proc Natl Acad SCi US A 2004, 101:14144-14149.

75. Yamada R, Nakatani Y, Ogino C, Kondo A: Efficient direct ethanol production from cellulose by cellulase- and cellodextrin transporter-coexpressing Saccharomyces cerevisiae. AMB Express 2013, 3:34.

76. Pridham TG, Raper KB: Ashbya gossypii - Its significance in nature and in the laboratory. Mycologia 1950, 42:603-623.

77. Mickelson MN: The metabolism of glucose by Ashbya gossypii. J Bacteriol 1950, 59:659-666.

78. Ribeiro O, Domingues L, Penttilä M, Wiebe MG: Nutritional requirements and strain heterogeneity in Ashbya gossypii. J Basic Microbiol 2012, 52:582-589.

79. Dietrich FS, Voegeli S, Kuo S, Philippsen P: Genomes of ashbya fungi isolated from insects reveal four mating-type Loci, numerous translocations, lack of transposons, and distinct gene duplications. G3: Genes, Genomes, Genet 2013, 3:1225-1239.

80. Ribeiro O: Physiological characterization of Ashbya gossypii and strain development for recombinant protein production. PhD thesis. University of Minho, School of Engineering; 2012.

81. Coleman ST, Fang TK, Rovinsky SA, Turano FJ, Moye-Rowley WS: Expression of a glutamate decarboxylase homologue is required for normal oxidative stress tolerance in Saccharomyces cerevisiae. J Biol Chem 2001, 276:244-250.

82. Kato T, Park EY: Expression of alanine:glyoxylate aminotransferase gene from Saccharomyces cerevisiae in Ashbya gossypii. Appl Microbiol Biotechnol 2006, 71:46-52.

83. McNeil JB, Zhang F, Taylor BV, Sinclair DA, Pearlman RE, Bognar AL: Cloning, and molecular characterization of the GCV1 gene encoding the glycine cleavage T-protein from Saccharomyces cerevisiae. Gene 1997, 186:13-20.

84. Albers E, Gustafsson L, Niklasson C, Lidén G: Distribution of 14C-labelled carbon from glucose and glutamate during anaerobic growth of Saccharomyces cerevisiae. Microbiology 1998, 144:1683-1690.

85. Wendland J, Walther A: Genome evolution in the eremothecium clade of the Saccharomyces complex revealed by comparative genomics. G3: Genes, Genomes, Genet 2011, 1:539-548.

86. Hébert A, Casaregola S, Beckerich JM: Biodiversity in sulfur metabolism in hemiascomycetous yeasts. FEMS Yeast Res 2011, 11:366-378.

87. Gottlieb D, Heideman W, Saba JD: The DPL1 Gene Is Involved in Mediating the Response to Nutrient Deprivation in Saccharomyces cerevisiae. Mol Cell Biol Res Commun 1999, 1:66-71.

88. Kim S, Fyrst H, Saba J: Accumulation of phosphorylated sphingoid long chain bases results in cell growth inhibition in Saccharomyces cerevisiae. Genetics 2000, 156:1519-1529.

89. Hiltunen JK, Mursula AM, Rottensteiner H, Wierenga RK, Kastaniotis AJ Gurvitz A: The biochemistry of peroxisomal beta-oxidation in the yeast Saccharomyces cerevisiae. FEMS Microbiol Rev 2003, 27:35-64

90. Kunau $\mathrm{WH}$, Dommes $\mathrm{V}$, Schulz $\mathrm{H}$ : beta-oxidation of fatty acids in mitochondria, peroxisomes, and bacteria: a century of continued progress. Prog Lipid Res 1995, 34:267-342.

91. Buston HW, Kasinathan S: The accessory factor necessary for the growth of Nematospora gossypii. III. The preparation of concentrates of the second accessory factor. Biochem J 1933, 27:1859-1868. 
92. Phalip V, Kuhn I, Lemoine Y, Jeltsch JM: Characterization of the biotin biosynthesis pathway in Saccharomyces cerevisiae and evidence for a cluster containing $\mathrm{BIO5}$, a novel gene involved in vitamer uptake. Gene 1999, 232:43-51.

93. Lopez F, Leube M, Gil-Mascarell R, Navarro-Aviñó JP, Serrano R: The yeast inositol monophosphatase is a lithium- and sodium-sensitive enzyme encoded by a non-essential gene pair. Mol Microbiol 1999, 31:1255-1264.

doi:10.1186/1471-2164-15-810

Cite this article as: Gomes et al:: Genome-wide metabolic re-annotation of

Ashbya gossypii: new insights into its metabolism through a comparative analysis with Saccharomyces cerevisiae and Kluyveromyces lactis. BMC

Genomics 2014 15:810.

\section{Submit your next manuscript to BioMed Central and take full advantage of:}

- Convenient online submission

- Thorough peer review

- No space constraints or color figure charges

- Immediate publication on acceptance

- Inclusion in PubMed, CAS, Scopus and Google Scholar

- Research which is freely available for redistribution 